\title{
Prof. Bruno Borghi
}

\author{
(in memoriam)
}

Bruno Borghi nacque in Milano il 20 luglio 19oo. Comp̀̀ gli studi di Medicina presso l'Università di Pavia, dove si laureò il i 8 luglio I924. Negli anni 1923-24 dovendo condurre a termine alcune ricerche sopra il quadro anatomo-patologico della tabe dorsale e della tabo-paralisi fu inviato dal Prof. Monti presso l'Istituto neurobiologico «A. Verga» di Mombello diretto allora dal Prof. Cerletti. Tali ricerche si concretarono in un'estesa relazione presentata come dissertazione di laurea.

Nell'ottobre del I 924 entrò come assistente effettivo nell'Istituto di Anatomia umana normale diretto dal prof. Livini. Nel dicembre del I925 fu designato, in seguito a concorso, a coprire il posto di Aiuto presso l'Istituto di Patologia generale dell'Università di Milano diretto da Pietro Rondoni. Nel febbraio del $193^{\circ}$

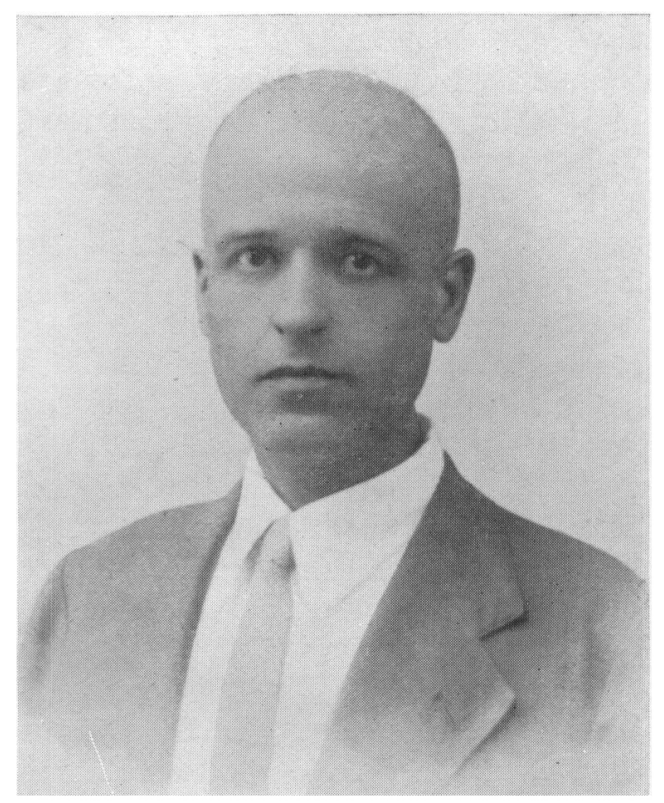
conseguì la libera docenza in Patologia generale. Nel I93 I, in seguito al conferimento di una borsa di studio della Rockfeller Foundation, si recò a Berlino presso la sezione delle Malattie tropicali dell'Istituto R. Koch diretta dal prof. Schilling. I lavori compiuti durante tale periodo furono ritenuti degni del premio d'incoraggiamento della fondazione Cagnola. Durante la sua permanenza a Berlino fu ospite del prof. Fischer al Kaiser Wilhelm Institut dove apprese le tecniche fondamentali delle culture "in vitro". Nel I934, in seguito a concorso, venne chiamato a reggere la Cattedra di Patologia generale dell'Università di Perugia, da dove nel r 936 si trasferì a Firenze.

Nel i944, durante i burrascosi anni che seguirono il passaggio del fronte e il ristabilirsi delle istituzioni democratiche nel Paese, fu assessore all'igiene del Comune di Firenze, carica che gli permise di mostrare le sue notevoli qualità tecniche e le non comuni doti di cuore. Dal 1947 al I 953 ininterrottamente fu Rettore dell'Ateneo fiorentino, carica che ricoprì con dignità e con decoro affatto personali. Per i suoi meriti di Scienziato e di Uomo venne insignito della Legion d'onore.

Le ricerche del Borghi sono state rivolte a vari campi della fisiopatologia. Un 
gruppo di ricerche riguarda l'influenza dei carboidrati sui processi rigenerativi e alcune particolarità del ricambio di questi prodotti. L'influenza del glucosio in alte dosi è negativa sui processi riparativi del coniglio, mentre favorisce quelli del tritone, nel coniglio si nota anche una diminuzione del glicogeno epatico, turbe di rilievo si notăno anche in animali tenuti a dieta scorbutigena. Di notevole interesse sono le ricerche del Borghi nel campo dell'oncologia sperimentale: fra i primi accennò all'importanza dell'ospite nell'insorgenza della proliferazione tumorale, mettendo in evidenza un movimento del S.R.E. inversamente proporzionale alla crescita della neoplasia e cioè che tumori con notevole tendenza alla crescita ed all'infiltrazione suscitano nell'ospite uno scarso movimento istiocitario, il contrario, verificandosi in tumori a scarso sviluppo. Fra i primi riusci ad intravedere una certa deviazione del metabolismo degli zuccheri nei tessuti neoplastici, dati che furono, successivamente, confermati da altri AA. Numerose le ricerche in campo microbiologico riguardanti le tripanosomiasi, i prodotti di autolisi batterica, da cui riusci ad isolare un principio ipertermizzante ed un principio favorente la leucocitosi, l'attività fosfatasica del bacillo tubercolare.

Di singolare rilievo sono le ricerche del Borghi e della sua Scuola sul ricambio proteico della cute. Egli, oltre alla dimostrazione di particolari via metaboliche per alcuni aminoacidi in tale tessuto, intravide la possibilità, che ricerche successive di altri AA. hanno pienamente confermato, che le sintesi proteiche iniziassero da composti estremamente semplici. Era intenzione negli ultimi anni di condurre una serie di ricerche miranti ad indagare quale parte tali vie sintetiche avessero nella crescita tumorale, ma purtroppo la repentina scomparsa ha troncato questa sua aspirazione.

Per caratterizzare la Sua figura scientifica non è fuori luogo citare il giudizio del Suo grande Maestro: «Io devo soprattutto apprezzare l'onestà scrupolosa del giovane ricercatore, la quale lo ha sempre tenuto lontano da una produzione voluminosa, affrettata e superficiale dai risultati incerti e caduchi; ed il grande ed equilibrato fervore, che non è mai degenerato in pericoloso facilonismo ed in una corsa sterile e deplorevole dietro agli argomenti e tecniche di moda ».

Del Borghi vogliamo in questa sede ricordare il favore con il quale sempre considerò gli studi della genetica derivato a Lui dallo spirito del Suo grande Maestro il Prof. Pietro Rondoni. Anche la nostra Rivista ne recò una significativa testimonianza l'anno scorso quando Bruno Borghi volle in un lungo ed autorevole articolo, recensire il nostro volume sul novantesimo delle leggi mendeliane. Ne ricaviamo un brano che ha tanto maggior valore in quanto dettato dall'Ordinario di Patologia Generale nell'Università di Firenze:

«Le Genetica applicata alla Patologia è sicuramente una luce nuova potenziale, che riapre il capitolo dell'etiologia non tanto per accogliervi episodicamente conoscenze nuove scaturite da ricerche isolate e frammentarie, quanto piuttosto per rivedere alla luce della Genetica il significato più preciso dei fattori organismici di malattia, in rapporto alla loro eventuale natura di fattori aventi rapporti più o meno stretti con l'eredità biologica.

La possibilità di applicare la Genetica alla stregua di metodo per l'esecuzione 
di ricerche che interessano il caso di malattia, vale a dire la possibilità di avviare un indirizzo di ricerca clinica basantesi sopra leggi naturali valide per tutti i viventi, uomo compreso, sotto quale punto di vista potrà giovare alla clinica? In linea generale si potrebbe rilevare che il giovamento sarà simile a quelli offerti generalmente da ogni e qualsiasi mezzo mediante il quale il medico conosce il proprio paziente: la Genetica Medica però offre al medico possibilità di conoscere il paziente nella sua individualità, fatto assai importante agli effetti non soltanto diagnostici. Conoscere sufficientemente l'individualità del paziente, significa infatti conoscere anche i fattori di natura ereditaria, suscettibili di interferire sui processi morbosi e di imprimere - insieme ad altri fattori coesistenti di natura ambientale - determinate varianti di decorso, di evoluzione e di risoluzione dei processi morbosi. Ne gioverà sicuramente la diagnosi dal punto di vista dell'esattezza, ma ne gioverà pure la Terapia, che rappresenta la finalità ultima dell'azione del medico.

Nel concludere la segnalazione di un'opera pubblicata in omaggio alla memoria di Gregorio Mendel, i cui meriti scientifici sono quelli di uno studioso al quale l'Umanità è debitrice di una scoperta di portata universale nel mondo dei viventi, il pensiero corre verso altre memorie e verso altre ricerche, dalle quali - per merito di De Giovanni e di Viola - nacque la Scienza delle Costituzioni, basata sull'aspirazione di riuscire a valutare con sufficiente esattezza i fattori organismici dei pazienti e di dedurne tutte le possibili conseguenze a vantaggio dei pazienti stessi. Limitatamente all'uomo, veniamo a trovarci di fronte a direttive di indagini e di applicazioni destinate ad incontrarsi e ad integrarsi vicendevolmente. Il genotipo della stirpe umana, tanto auspicato dalla Scuola Costituzionalistica Italiana onde giungere alla conoscenza effettiva del fenotipo, si presenta agli occhi degli studiosi contemporanei come un voto, nella successione delle nostre conoscenze che sarà colmato dalla Genetica Clinica, la quale rappresenterà l'integrazione delle indagini cliniche aventi l'obbiettivo di conoscere il malato. Una applicazione delle Leggi dell'Eredità Biologica così elevata e così degna, perchè di giovamento al sofferente, è la migliore testimonianza di ammirazione che Gregorio Mendel potrà avere in un prossimo futuro, che si auspica prossimo assai ".

L. GEDDA 